\title{
Proceedings of the joint Meeting of the Association of British Neurologists and Spanish Society of Neurology, Valencia, Spain, 1990
}

CENTRAL LESIONS IN NEUROFIBROMATOSES: 30 CASES WITH A PROPOSAL FOR A NEW

CLASSIFICATION

A Pou-Serradell, A Ugarte-Elola. Barcelona, Spain

The National Institutes of Health Consensus Panel on neurofibromatosis (NF) recognised two distinctive forms (NF-1 and NF-2) and stated that variant forms may exist. The authors selected 30 patients that fulfilled diagnostic criteria for NF-1 or NF-2, all with MRI and histopathological confirmation in 19 cases. Nineteen patients presented exclusively with intraparenchymatous lesions in the CNS. All 19 fulfilled a diagnostic criteria of NF-1. Histopathological study demonstrated, in the eight cases submitted for examination, pilocytic astrocytomas (six cerebral and two optic nerve). Ten patients showed only extra-axial lesions; two of them fulfilled diagnostic criteria for NF-1 and the histopathological study showed neurofibromas; eight patients fulfilled diagnostic criteria for NF-2 and at histological examination there were schwannomas $(7 / 8)$ or ganglioneuromas $(1 / 8)$. One patient had intra and extra-axial lesions and had partially diagnostic criteria for NF-1 and NF-2. Pilocytic astrocytomas appear to be the specific lesions of the CNS for NF-1 patients. In these, the only extra-axial lesions are neurofibromas, and not acoustic. Schwannomas and meningiomas appear to be the specific lesions in NF-2; multiple spinal schwannomatosis, peripheral schwannomatosis and ganglioneuromatosis appear as variant forms of NF-2. Sporadic cases of NF-2 are much more common than hitherto suspected. It appears that more than two forms, and subdivisions, in NF-2 must be considered. A mixed form of neurofibromatisis, or NF-3, with specific lesions of NF-1 and NF-2 is rare.

SIMULTANEOUS PATHOLOGY IN THE INTERNAI AND EXTERNAL CAROTID ARTERY SYSTEM. A PROSPECTIVE CLINICAL AND

ANATOMOPATHOLOGICAL STUDY

F Asin Morales, JM Asin Morales, J Navarro Soria, LJ Lopez de Val, LF Pascual Millan, E Mostacero Miguel. Zaragoza, Spain

Two cases have been observed (a 35 year old man with Moya-Moya disease, and a 73 year old woman with ophthalmic herpes zoster and associated vasculitis) with simultaneous pathology in the internal and external carotid artery system. Both patients had severe narrowing in the internal carotid artery and biopsy of the superficial temporal artery showed proliferation without other pathological or inflammatory changes in the intima or adventitia.

To discover if this pathology was coincidental we carried out a prospective study with biopsies of the superficial temporal artery (external carotid system) on 13 patients with cerebrovascular diseases of different aetiologies, and also the internal carotid on 12 cases at necropsy. The authors found only atheromatous lesions in the carotid siphon in eight cases without clear correlation with the external carotid pathology. The temporal artery biopsies showed myofibrillar proliferation that had been divided into three grades of intensity; the pattern of medial enlargement by myofibrillar proliferation is probably a common response to different pathogenetic agents.

Biopsy of the superficial temporal artery could be of interest in those cases in which arteriography shows lesions of large and medium sized intracranial vessels.

\section{EARLY ONSET OF PARKINSON'S DISEASE IN 92} CASES

J Chacon, MA Pino, I Perez, M Pastor, JA Burguera, J Catala, M Aguilar, M Calopa, J Acosta. Seville, Malaga, Valencia, Barcelona and Cadiz, Spain

All patients with early onset parkinsonism with initial symptoms (less than 40 years of age) assessed by a neurologist in our study centres were studied, except those with secondary parkinsonism. In each case CAT and MRI were used

The 92 patients were divided into two groups according to age of onset of illness. Group 1 (onset $21-40$ years) $\mathbf{N}=86$ (males 52, females 34). Group 2 (onset before 21 years), $N=6$ (males 6 ).

There was a predominence of males in both groups (56-52\%/100\%). In Group 1, akinesia and rigidity predominated, while in Group 2 it was found that tremor predominated. The initial form in Group 1 was foca (88-37\%), Group 2 showed no predominance between focal or generalised forms ( $50 \%)$. Nine patients presented with dystonia as the initial symptom of the illness. In Group 1 the number of hereditary cases was remarkablefive families with affected siblings with early onset of parkinsonism and other different degree family members. This finding was not noticeable in Group 2. The delay in initiation of levodopa in Group 2 was considerably superior (16-16 years) than in Group 1 (4-63 mean years). The mean duration of treatment with levodopa in Group 2 was greater than that of Group 1 (13-5 mean years over 7-65). On the contrary, the present levodopa dose was less in Group $2(465 \mathrm{mg} / \mathrm{d})$ than Group 1 $(607-42 \mathrm{mg} / \mathrm{d})$. The majority of patients from both groups presented with fluctuations and dyskinesias.

A CONTROLLED STUDY OF MEMORY IN MYASTHENIA

T Del Ser, I Iriarte, J Domingo. Madrid, Spain

The assessment of memory function that is subserved by cholinergic neuronal systems may be a way of testing if there is central nervous system involvement in myasthenia gravis. We have studied 11 myasthenic patients (10 generalised and one ocular), nine neuromuscular patients (four rheumathoid arthritis, two systemic lupus erythematosus, one limb girdle myopathy, one chronic polyradiculoneuritis, one progressive sys- temic sclerosis) and 14 healthy controls, of similar sex, age and sociocultural background. Myasthenic patients were on chronic treatment with individually adjusted doses of corticosteroids (eight) or anticholinesterases (two) and five had had thymectomy. The following tests were applied: ipsimodal and transmodal reaction time, a divided attention task, attention span, sustained attention, iconic memory, short term memory in the Sternberg's paradigm, free recall, recognition, learning and delayed recall of words, verbal fluency, Rey's figure, WAIS subtests of vocabulary and similarities and the Beck's depression, the State-Trait Anxiety and the Bennet-Levi's Subjective Memory questionnaires. The myasthenic patients performed in these tasks as well as controls, and sometimes significantly better than neuromuscular patients. Only in one of the memory tasks were myasthenic patients worse than controls: they needed significantly more trials to learn a list of words. Our data agree with those previously reported, and suggest that well treated myasthenic patients do not have cognitive defects.

CORRELATION BETWEEN MRI AND CLINICAI

STATE IN 49 PATIENTS WITH MULTIPLE

SCLEROSIS

F Pujadas, J Sumalla, $M$ Molins, $M D$ de la Calzada, A Codina. Barcelona, Spain

MRI is very useful in the diagnosis of multiple sclerosis (MS). Nevertheless, the relationship between MRI abnormalities and the degree of clinical disability and course of the disease has shown contradictory results.

Forty nine patients with MS were reviewed, classified according to McAlpine's diagnostic criteria, into possible (nine cases) probable (13 cases), and definite (27 cases). Age, duration of disease, clinical course (relapsing/remitting, progressive or combined), number of relapses, and degree of clinical disability using the Neurological Rating Scale (NRS) of Sipe et al were recorded. Severity of MRI abnormalities was evaluated using the scale proposed by Stevens $e t a l$, based on the number and size of lesions showed by MRI.

Mean age of patients was 35.2 years (SD $=11.6$; range: 17 to 68 ). Duration of disease varied from two months to 31 years [mean (SD) $6.6(6.8)$ years]. The degree of clinical disability according to NRS was between 44 and 100 points [mean (SD) $78 \cdot 1$ (16)]. One patient had MRI grade 1, eight patients MRI grade 2, 18 patients MRI grade 3, 15 patients MRI grade 4, and seven patients MRI grade 5. MRI involvement showed a moderate correlation with McAlpine's criteria $(r=+0.4282, p<0.01)$ and lower but significant correlation with clinical disability according to NRS $(r=-0.3392, p<0.05)$ and number of relapses $(r=+0.3457, p<$ $0.05)$. No significant correlation with age $(r=+0.0933)$, duration of disease $(r=+0.1957)$, or clinical course were observed. 
SUBACUTE SENSORY NEURONOPATHY AND SMALL CELL LUNG CARCINOMA: DIAGNOSTIC USEFULNESS OF ANTI-HU ANTIBODY

R Reñé, F Graus, X Ferrer, F Miralles, J Dalmau, JA Martinez Matos. Barcelona, Spain and New York, USA

Two patients with small cell lung carcinoma who developed a dysaesthesiae, paraesthesiae, severe impairment of vibratory and postural sensibility, sensory gait ataxia and universal areflexia suggesting paraneoplastic subacute sensory neuronopathy (PSSN), are reported. In one patient PSSN appeared three months before the diagnosis of carcinoma, and simultaneously with the diagnosis in the other. Both patients had cancer with limited metastatic dissemination.

Polyclonal Ig G (anti-Hu) against neuronal nuclei was detected in sera from both patients using indirect immunofluorescence, indirect immunoperoxidase, and avidin-biotin immunoperoxidase methods. Western-blot analysis confirmed the existence of anti-Hu antibody in both sera, identifying a nuclear neuronal antigen with a molecular weight of 35 to $40 \mathrm{KDa}$.

CAN JUVENILE MYOCLONIC EPILEPSY OF JANZ SEEM SIMILAR TO AN "INTRACTABLE" EPILEPSY?

J Salas-Puig, A Tunon, V Mateos, LM Guisasola, CH Lahoz. Oviedo, Spain

The authors studied 54 patients with juvenile myoclonic epilepsy of Janz (JME) and analysed six patients who had, for a mean of $15 \cdot 5$ (8-34) years, a poor response to polytherapy. For many years all had daily myoclonic seizures, several myoclonic status episodes, frequent tonic-clonic seizures in five patients psychiatric disturbances. The diagnosis of JME was made and sodium valproate achieved a good control in the majority of them. These patients were compared with the other patients of our series who had not had a severe evolution. There are no neurophysiological differences between them. A long diagnostic interval (12-31 years), daily myoclonic seizures, other types of seizures (absences, tonic-clonic, clonic-tonic-clonic), photosensitivity and/or psychiatric disturbances do not suggest a poor response to adequate treatment. Sodium valproate is the treatment of choice for JME.

THE SET TEST AS A DIAGNOSTIC MARKER OF ALZHEIMER'S DISEASE

LF Pascual Millan, JV Martinez Quinõnes, TES Sanchez Valien, J Modrego Pardo, JA Mauri Llerda, LJ Lopez del val, E Mostacero Miguel, F Morales Asin. Zaragoza, Spain

Aphasia is an important diagnostic criterion of dementia of the Alzheimer type (DAT). Since word list generation and naming are language functions that are most abnormal, a rapid evaluation of verbal fluency and naming could be a useful diagnostic method in DAT patients.

The Set Test is a rapid category verbal fluency test. The subject has to name as many items as he can recall in each of four successive categories or sets: colours, animals, fruits and towns. One point is awarded for each correct item offered, with a maximum of 10 in each set and a maximum total score of 40 . Isaacs and Akhtar (1972) obtained a mean score of $31 \cdot 2+7 \cdot 3$ in healthy old people ( $n=189$ ).

The authors studied 41 patients with DAT, 25 patients with multinfarct dementia (MID) and 12 patients with other dementias. The dementia severity was evaluated with the MEC (Spanish adaptation of the MMSE, with a maximum possible score of 35 ). The mean Set Test score in the DAT group was $17 \cdot 8$ (7.8); in the MID group was $30 \cdot 2(6 \cdot 5)$ and in the other dementias group was 25.6 (7) Pairing dementia groups by severity, in sligh dementia (MED 25-29) DAT patients had a mean Set test of $28 \cdot 6$. MID patients 32.4 and other dementias $31 \cdot 6$. In moderate dementia (MEC 15-24), DAT: 20.7; MID: 29.7; other dementias 24.4. In severe dementia (MEC <14), DAT: 14.5; MID\% 25.6. Other dementias: $16 \cdot 9$.

Only five patients in the DAT group (12\%) scored more than 25 points on the Set Test. These data demonstrate that for comparable dementia severity, DAT patients have the most profound category of naming deficit. A Set test score less than 25 points in a patient with slight or moderate dementia could be a useful diagnostic marker of DAT.

COGNITIVE DYSFUNCTION IN EPILEPSY. ROLE OF ANTIEPILEPTIC DRUGS AND OTHER FACTORS A DOUBLE-BLIND COMPARATIVE STUDY OF PHENYTOIN VERSUS CARBAMAZEPINE J Lominchar, J Morera, A Sanchez, M Garcia, JM Láinez, J Sancho, $R$ Yayá. Valencia, Spain

A study is presented comparing cognitive dysfunction in a group of 20 patients with primary epilepsy, and newly treated with antiepileptic drugs (AED), either phenytoin (11) or carbamazepine (9), using a monotherapy regime and compared with a control group of 10 normal patients without treatment. Only patients without cranial CT abnormalities and Weschler Adult Intelligence Scale (WAIS) performance above 80 were included. To study cognitive function each patient performed a neuropsychological battery test including the WAIS, Signoret's Battery 144, Luria's logical memory test, a simple motor velocity test, a visuomotor coordination test and a sustained attention test. The complete battery was passed to every patient just before the beginning of treatment, at four weeks, and at six months. The results were correlated with the number and control of fits, age and sex of patients and serum levels of AED determined just after the battery test.

No significant difference was found between the group treated with phenytoin and the group treated with carbamazepine in any of the neuropsychological tasks evaluated.

A significant improvement $(p<0.01)$ was found in the treated group versus the controls in memory retention, verbal memory efficiency and in deferred verbal recall tasks.

Patients in whom epilepsy began before age 15 performed worse in the simple motor velocity and visuomotor coordination tasks.

High serum levels of AEDs-even in the normal range-correlated well with difficulties in the visual and memory learning tasks and with a forgetfulness tendency, while low serum levels of AEDs and controlled epilepsy (no fits) correlated with better results in the attention and memory subtests.

Although it is thought that cognitive dysfunction in epileptics can be due to many factors, the findings suggest that AEDs are one of them, although we have found no differences between the two drugs tested, together with seizure control and the age of onset of epilepsy.

AN EXPERIMENTAL MODEL OF PAINFUL NEUROMA: ANALYSIS OF ELECTRICAL ACTIVITY J Laguarda, J Sancho, JM Láinez, J Barberá. Valencia, Spain

Sectioning of the sciatic nerve alters behaviour in the rat, reflected by auto-mutilation of the denervated limb (autotomy). Wall et al (1979) interpreted autotomy as an expression of chronic pain on the part of the animal, due to abnormal impulses generated within the resulting neuroma. The authors showed that prolongation of the proximal stump of the sectioned nerve employing a fascicular graft delays the appearance of autotomy for a period of time proportional to the length of the graft.

The sciatic nerve was sectioned (with or without fascicular grafting) in $\mathbf{3 0}$ wistar rats. In groups of two animals each, we investigated spontaneous proximal activity in the distal stump of the sectioned nerve or graft after 5, 7, 10, 14, 21, 28 and 35 days. A clear difference was observed in the appearance of spontaneous electrical activity. Whereas in rats presenting neuroma electrical activity appears on the seventh day, those animals subjected to grafting show activity only after day 40. This agrees with the increase and evolution of the degree of autotomy already observed previously. These observations confirm the theory that automutilation is related to the electrical activity of the regenerated fibres within the neuroma, and lends support to the use of this animal model of chronic pain.

\section{COMBINED TREATMENT WITH PLASMA} EXCHANGE AND HIGH DOSE INTRAVENOUS IMMUNOGLOBULIN IN MYASTHENIA GRAVIS

J Tejada, E Diez-Tejedor, A Frank, M Lara, P Barreiro. Madrid, Spain

The authors report the results in the combined therapy of plasma exchange and highdose intravenous gamma globulin in four patients with myasthenia gravis (three of them in Osserman's grade II-B and one in III). They started with plasma exchange and continued with intravenous infusion of Immunoglobulin (400 $\mathrm{mg} / \mathrm{Kg}$ weight/day) for five days. A patient classification system based on the University of Virginia's modification of the Osserman classification and a scale of activity was adapted to grade response to therapy. All patients showed an improvement in their functional status from $4 \cdot 2(0 \cdot 81)$ (moderate to severe generalised weakness) to $2 \cdot 2(0.9)$.

The improvement started between the first and the sixth day after administration of Immunoglobulin. The time of maximal improvement was between the second and fourth weeks. One of these patients received this therapy prior to thymectomy.

It is suggested that combined treatment with plasma exchange and intravenous Immunoglobulin may increase their immunological effects: plasma exchange in decreasing AChR antibody title and intravenous Immunoglobulin in limiting ACh destruction by interfering with modulation and the complement cascade. 
DEPRESSION IN PARKINSON'S DISEASE AND ITS POSSIBLE RELATION WITH MOTOR AND COGNITIVE MANIFESTATIONS

D Gil-Saladié, M Delgado, C Oliveras-Ley, J Peña, Ll Barraquer-Bordas. Barcelona, Spain

The authors examined the relationship between depression and motor and cognitive deficits in 30 non-demented patients with Parkinson's disease (PD). There were 19 men and 11 women, average age $60 \cdot 2(7 \cdot 7)$ years, and average schooling $7.5(2.5)$ years. A neuropsychological battery (PIENCBarcelona '86) was used to compare this group of PD subjects with a second group of 52 healthy subjects ( 27 men, 25 women) of average age $55.8(12.9)$ years, and average schooling $8.6(3.5)$ years.

This battery explored orientation, language, praxis, gnosis, attention and concentration, memory and WAIS type intelligence tests. There was a significant ( $p \leqslant 0.01$ ) difference between the two groups on 57 out of 144 subtests.

The authors also applied a geriatric depression scale to the PD group. Either $70 \%$ or $50 \%$ of the patients were considered depressed depending on the cut-off score. These scores on the depression scale were not correlated to the results on the 57 neuropsychological subtests for which a group difference from controls had been demonstrated. In addition, there was no correlation between the depression score and the numerical Hoehn and Yahr rating.

Despite the absence of dementia, the Parkinsonian patients performed significantly worse on a number of the subtests used on the neuropsychological battery. In addition, there was a high prevalence of elevated scores on the depression scale used. However, there was no relationship between depression, impairment on cognitive subtests and overall motor impairment. This suggests the possibility that depression in PD is due to a neurochemical disturbance which does not significantly affect cognitive or motor functions.

AUDITORY FUNCTION IN

FACIOSCAPULOHUMERAL DYSTROPHY

JJ Vilchez, H Pérez-Garrigues, J Catalá, J

Marcote, B Casanova, T Sevilla. Valencia, Spain

Facioscapulohumeral dystrophy (FSHD), is an autosomal dominant inherited disease characterised by marked variability in the expression and intensity of symptoms Several cases of FSHD with hearing loss, associated or not with vascular retinal abnormalities (Coats' syndrome), have been described either sporadically or in several members of the same family. The authors have studied the auditory function in a group of FSHD with the aim of detecting the prevalence of hearing loss in this disease.

The study was carried out on 33 patients (11 males, 22 females) of 10 families with FSHD. Eighteen of them were affected, and 15 were asymptomatic relatives. None of them had previous history of known causes of deafness. Auditory threshold was obtained by testing bone and air conduction with an audiometer, Interacoustic AC-4, and headphone TDH-39. $125 \mathrm{~Hz}$ to $8000 \mathrm{~Hz}$ frequencies were tested. The auditory function in al healthy relatives was normal. Six patients $(33.3 \%)$ showed a sensorineural hearing loss, two of them being members of the same family. One case showed unilateral hypoacusis and the rest were bilaterally affected. Two of the hypoacusic patients manifested a severe form of FSHD, two were affected by an intermediate form and the other two showed minimal symptoms.

The significant prevalence of hypoacusis demonstrated in this series of FSHD compared with the asymptomatic relatives $(p<$ 0.05 ) indicates that auditory abnormalities form an important part of the FSHD picture. It is considered that this manifestation could have a variable prevalence. The hypoacusis does not seem to be related to the intensity of the muscular symptoms. The pathogenesis of this abnormality is unknown.

DRUGS INDUCING PARKINSONISM IN OUR ENVIRONMENT

JF Marti Massó, N Carrera, M Urtasun, A López de Munain. San Sebastián, Spain

Parkinsonism induced by drugs (PID) is the second most common cause of the parkinsonian syndrome, idiopathic Parkinson's disease (PD) being more common. The authors present two groups of PID, studied in the last seven years, one of them retrospectively (72 patients), and the other one prospectively (21 patients). The clinical picture in PID patients is similar to PD patients. Simultaneous pos tural and rest tremor, subacute bilateral onset, buccolingual dyskinesia before antiparkinsonian treatment and slow progression of the disease are suggestive of PID. The most common drug inducing parkinsonism in both series was cinnarizine. About $30 \%$ of the patients were taking more than one offending drug. Recovery after drug withdrawal was complete in a mean period of 9.5 months in akinetic-rigid patients, and 14.6 months in bilateral tremor syndrome. After an asymptomatic period of time, six out of 72 retrospectively studied patients, developed PD, and another three Progressive Supranuclear Palsy. Clebopride, aprindine and buphormine were encountered as causes of PID for the first time.

DETERMINATION OF LANGUAGE DOMINANT HEMISPHERE WITH RAPID TRANSCRANIAL MAGNETIC STIMULATION (rTMS) Alvaro Pascual-Leone G, John R Gates, Ani K Dhuna. Minneapolis, MN, USA

Hemispheric dominance for language in patients undergoing evaluation for epilepsy surgery is currently determined with a WADA test. Attempts to induce speech arrest using non-invasive transcranial magnetic stimulation (TMS) with stimulation frequencies of up to $5 \mathrm{~Hz}$ have been unsuccessful. The development of a rapid TMS (rTMS) capable of stimulating the cortex at rates similar to those used for direct cortical stimulation raised the possibility of noninvasive localisation of language.

Twelve adult epileptic patients had rTMS over the left, then over the right hemisphere by investigators "blind" to the patients" handedness. The stimulation coil, a water cooled round coil of $11 \mathrm{~cm}$ in diameter, was centred successively over P3(4), C3(4), F3(4), and $F 7(8)$. At the stimulation point the coil was held in a sagittal plane and angulated towards the vertex so that it lay flat on the scalp. rTMS was initially at $40 \%$ of maxima output intensity and frequency of 8,16 , and $24 \mathrm{~Hz}$. Stimulation at each point was perfor- med for 10 seconds while the patient was asked to count aloud. The endpoint was the induction of speech arrest. If no speech arrest was induced the stimulus intensity was increased to $60 \%$, and later to $80 \%$. During the stimulation the patients were continuously monitored with video-EEG and ECG.

Stimulation at F7 and F8 induced tremulous, dysarthric speech in all patients probably due to facial muscle contractions. But in 11 of the 12 subjects a definite interhemispheric difference was documented, in that stimulation over F7 lead to reproducible, complete speech output interruption. This finding was interpreted as localising language dominance to the left hemisphere in these 11 patients. The results were confirmed by the WADA test. In the one patient where right and left hemispheric stimulation allowed no differentiation, the WADA test demonstrated a bihemispherically shared language dominance. Our results support the hypothesis that a technique can be developed for noninvasive determination of hemispheric dominance for language.

INDUCTION OF SEIZURES AND ACTIVATION OF EPILEPTIC FOCI WITH RAPID TRANSCRANIAL MAGNETIC STIMULATION (rTMS)

Anil K Dhuna, John R Gates, Alvaro Pascual-Leone G. Minneapolis, MN, USA

Initial studies of transcranial magnetic stimulation (TMS) in epileptic patients failed to induce seizures or trigger epileptiform activity in the EEG. Recently though, Hufnagel et al described specific activation of the epileptic focus in 12 of 13 patients with medically intractable epilepsy using TMS at $0.05-0.5 \mathrm{~Hz}$. They were unable to induce epileptiform potentials outside the epileptic foci, which had been identified by corticographic recordings, and suggested that TMS could be a useful tool for localisation of epileptic foci in presurgical evaluation.

Using rTMS at $16 \mathrm{~Hz}$ and $80 \%$ maximal output intensity we were able to induce a focal seizure from the mid temporal area contralateral to the known epileptic focus in a patient undergoing evaluation for epilepsy surgery. Conversely, we have been unable to trigger seizures or induce epileptiform discharges arising from the epileptic focus in any of 12 patients using rTMS at $8-24 \mathrm{~Hz}$ and 40 $80 \%$ maximal output intensity. Thus rTMS failed to activate the patients' usual epileptic focus while inducing a focal seizure in the contralateral hemisphere in one patient. These preliminary data suggest that the epileptogenic potential of TMS may be dependent on the frequency of stimulation.

THERMOGRAPHY AND THE DIAGNOSIS OF CARPAL TUNNEL SYNDROME

WN Macleod, S Wilson. Ninewells Hospital, UK

Thermography is used in some centres to diagnose nerve compression syndromes. We have studied the validity of this practice. We investigated the autonomic control of the hand vasculature using a thermographic scanner (Phillips Thermoscan) and a lase Doppler flow (LDF) meter (Perimed, PF2b), in seven patients with electrophysiologically verified unilateral median nerve (MN) compression. Three of the nerves had absent median sensory action potentials and marked wasting of abductor pollicis brevis. All cases had ongoing nocturnal numbness waking. 
Subjects were brought into states of peripheral vasoconstriction and vasodilatation, evidenced by the thermographic appearance of the hands and a static LDF recording from digit 5 , by seating them in an environmentally controlled box in a temperature controlled room. Using this qualitative dynamic testing, we looked for evidence of failure to vasoconstrict or vasodilate in the fingers and for temperature asymmetries in the hands.

All seven affected hands vasodilated normally with digits of both hands appearing symmetrically warm, often with the finger pulp warmer than the more proximal hand. They also vasoconstricted normally, showing a downward temperature gradient from palm to finger tips. LD measurements of basal digital cutaneous flow levels and the reflex responses to a cold challenge to the foot confirmed these findings. On re-warming after maximal vasoconstriction asymmetries exceeding $3^{\circ} \mathrm{C}$ between the symptomatic and contralateral hands were seen in two cases.

The authors found that the autonomic control of the peripheral vasculature mediated by these seven damaged median nerves was intact. Thermography is not a sensitive tool in the diagnosis of median nerve entrapment.

PATHOLOGICAL PATTERNS OF F-WAVE LATENCY DISPERSION IN ABDUCTOR POLLICIS BREVIS (APB)

WN Macleod, Dundee, UK

This study investigates the relationship of the minimal $\mathrm{F}$-wave latency ( $\mathrm{F}$ min) to the $\mathrm{F}$ wave latency range ( $F$ min-max) when the peripheral nerve supply is damaged. The electrodiagnostic sensitivity of the $\mathrm{F}$ minmax measurement is considered.

Thirty four consecutive hands (25 carpal tunnel syndrome (CTS), six peripheral neuropathy, two Guillain-Barré syndrome (GBS), one brachial plexopathy) with an electrodiagnostic median nerve lesion were included. Thirty F-waves were recorded from the surface of each APB (filters $20 \mathrm{~Hz}-2$ $\mathrm{KHz}$ ) using a supramaximal wrist stimulus. For each median nerve (MN) forearm segment motor conduction, distal motor latency (DML), antidromic sensory wrist-palm latency (over $8 \mathrm{~cm}$ ) and a mixed trans-wrist conduction velocity were recorded. In the analysis of data our laboratory control values for motor, sensory, mixed and $F$ min values (height matched) were used. Control $\mathrm{F}$ minmax values came from another study $(n=36$, same height range, mean $F$ min-max $=2.44 \mathrm{~ms}$, maximum $4 \mathrm{~ms}$ ).

In $18 \%$ of hands no $\mathrm{F}$-wave latency abnormality showed; only a sensory/mixed conduction defect. In $32 \%$, F min-max was abnormal (4-10 ms) while $F$ min was normal. In $24 \%$, F min-max was the only electro-diagnostic abnormality (sensory/mixed studies normal). In $32 \%$, F min was prolonged while $\mathrm{F}$ min-max was $\leqslant 4 \mathrm{~ms}$ (in two cases the DML was $>4.5, \leqslant 8 \mathrm{~ms}$ ). In only $18 \%$ of hands $\mathrm{F}$ min and $\mathrm{F}$ min-max both lay outside the control ranges.

The results show that the F-wave latency range is not uncommonly normal when the minimal F-wave latency is prolonged. In the nerves which showed no F-wave latency abnormality there may have been a pure sensory fibre lesion. It is noteworthy that $F$ min-max may be an isolated electro-diagnostic abnormality (CTS, GBS). F mix-max is a useful measurement in nerve conduction studies and, as shown elsewhere, its sensitivity can be further increased by using a needle rather than a surface electrode to record the F-wave onset.

\section{VISUAL CHANGES IN MIGRAINE}

\section{NJ Legg, NM Khalil. London, UK}

Psychophysical visual tests and VERs were studied in 90 migraine subjects and 60 controls. Contrast sensitivity was normal in common migraine but reduced in classical migraine of long duration, especially on the side of the visual aura. Illusions elicited by high-contrast gratings were more frequent in migraine, especially in classical migraine and on the aura side, but did not change with duration. ERGs were all normal. In pattern VERs the P100 latency was prolonged in all cases, and P100 amplitude was higher in common migraine, and in classical migraine of short duration, but lower in chronic cases. With single-flash VERs the $P_{1}$ and $P_{2}$ latencies were prolonged in all cases. With repetitive flash the $\mathrm{H}$ response was more frequent in all cases, especially in classical migraine of short duration. In patients who received successful prophylactic treatment the $H$ response and the illusions returned towards normal.

The increased illusions, prolonged VER latencies, high P100 amplitude and increased $H$ response all indicate an underlying visual abnormality in migraine subjects. The reduction of P100 amplitude, $H$ response and contrast sensitivity in late classical cases may represent ischaemic damage caused by recurrent attacks.

SEIZURE INDUCTION BY ALCOHOL IN PATIENTS WITH EPILEPSY. EXPERIENCE IN TWO HOSPITAL CLINICS

J Heckmatt, AA Shaikh, Adrienne Moffett, $M$ Swash, DF Scott, London, UK

Seizures may occur shortly after chronic heavy alcohol intake is abruptly discontinued. The importance of alcohol ingestion as a trigger factor for seizures in patients with chronic epilepsy is less well established. 'To study this further 70 epileptic patients were surveyed attending a general neurological clinic and 64 patients attending an epilepsy clinic, to determine the frequency of alcohol related seizures. Seven $(10 \%)$ of the neurological clinic patients, and nine $(15 \%)$ of the epilepsy clinic patients reported exacerbation of their seizures with alcohol. In the first group, two had been heavy drinkers when under age, two had features of alcohol dependence and three had experienced resolution of seizures following cessation of their drinking. In the second group five drank four units per day or more, and one drank much more heavily. The importance of alcohol in the causation of these patients' seizures had not been fully appreciated, a situation often confounded by abnormal liver function tests caused by anticonvulsant medication. Hence, enquiry about, and control of, alcohol ingestion is important in the management of patients with intractable seizures.

"FUNNY TURNS" AN AMBULATORY EEG AND FOLLOW UP STUDY

SJL Howell, LD Blumhardt. Liverpool, UK

Three hundred and sixteen patients suffering non-focal episodes of altered awareness undiagnosed after history and examination by a neurologist were monitored with simultaneous ambulatory cassette EEG and ECG two channels of EEG and one of ECG) Typical attacks were recorded in 64 patients and minor versions of typical attacks in a further 42. On masked review typical episodes were classified epileptic in 15 patients and minor episodes classified epileptic in two. To validate the diagnoses made on the basis of monitoring and to determine the cause of attacks in the remaining patients, a follow up study was undertaken. Follow up of greater than two years was obtained in 290 patients $(92 \%)$. Nineteen had died, 17 from causes apparently unrelated to their attacks. Of the remaining 271 patients (mean follow up 64 months), a firm diagnosis was made in 75 . In $40(15 \%)$ the attacks were diagnosed as epileptic, in 18 they were thought to be psychologically determined (including 10 pseudoseizures) and in two due to cardiac arrhythmias. Ambulatory EEG had originally been rated as normal during five attacks diagnosed as epileptic on follow up. One attack had been classified "equivocal-possibly epileptic" in a patient subsequently thought not to have epilepsy.

ELECTROPHYSIOLOGICAL STUDIES IN THYROTOXIC HYPOKALAEMIC PERIODIC PARALYSIS: THE EFFECT OF TREATMENT DA Ingram, J Monson, M Swash. London, UK

The effect of therapy was monitored in a 50 year old white male who presented with recurrent episodes of thyrotoxic hypokalaemic periodic paralysis (THPP). Interictal recordings from abductor digiti minimi following three minutes maximal voluntary contraction showed reduction of the maximal compound muscle action potential (CMAP) amplitude, reaching a nadir of $25 \%$ of the resting value at 2.5 hours. After eight hours the CMAP amplitude had recovered to only $60 \%$ of the resting value. In 10 normal control subjects and six hyperthyroid subjects without paralysis, the post-exercise CMAP was never less than $70 \%$ of the resting value. Conventional single fibre EMG and intramuscular microaxonal stimulation techniques in the patient revealed occasional blocking in association with normal jitter Selective stimulation and extracellular recording of individual muscle fibres in vivo revealed progressive reduction in size of the action potential spike until blocking occurred.

Following therapy with carbimazole and $I^{131}$ the electrophysiological abnormalities gradually improved, reaching normal values after three months. This occurred in paralle with the clinical improvement in muscle strength and subjective weakness.

The findings indicate that the essential abnormality in THPP is a generalised conduction defect in the muscle fibre membrane which resolves when the patient is rendered euthyroid.

MOTOR NEURON DISEASE IN LANCASHIRE AND SOUTH CUMBRIA 1976-86-A GEOGRAPHIC STUDY JD Mitchell, HN Gibson, AC Gatrell. Preston and Lancaster, UK

It is the subjective impression of many neurologists that motor neuron disease (MND) sometimes occurs in geographical clusters. An attempt to test this more objec- 
tively was made by making an analysis of the postcode of the place of residence of 171 patients who presented with MND from a population of approximately 1.8 million residents in Lancashire and South Cumbria, over a 10 year period. The postcode can specify the location of an address to within $100 \mathrm{~m}$. Two patients shared the same unit postcode (covering an average population of 38). Significant excesses of patients were found in a range of electoral wards which in some cases related to specific age ranges of disease onset. While statistical analysis of such data is always difficult, particularly for rare diseases such as MND, it seems increasingly likely that MND does not have a random geographical distribution.

Clustering of year of birth of MND patients was also sought. This has been reported to occur in Parkinson's disease. No evidence of such clustering was found in MND.

These findings give some support to current ideas that neurodegenerative diseases might be related to exposure to environmental factors.

APOMORPHINE AND PARKINSON'S DISEASE AJ Lees, J Frankel, A Hughes, N Turjanski, B Kleedorfer, GM Stern. London, UK

Apomorphine, a dopamine receptor agonist, was given subcutaneously to 67 levodopa treated patients with Parkinson's disease, who had refractory off-period disabilities. In 45 patients given intermittent suprathreshold injections by insulin pen for a median period of 28 months, the mean number of hours spent in a disabled state fell from $7 \cdot 2$ to $2 \cdot 7$. Similar benefit was obtained in 21 patients receiving continuous 24 hour subcutaneous infusions of apomorphine with bolus demands administered by an ambulatory mini pump. The incidence of long term neuropsychiatric side effects was low $(7 \%)$ and tachyphylaxis occurred to peripheral side effects such as nausea, vomiting and postural hypotension. Single subcutaneous injections of apomorphine have also been used as a test for dopaminergic responsiveness in 90 patients with the parkinsonian syndrome and correlated with responses to an oral levodopa challenge and chronic levodopa treatment. These results suggest that apomorphine may be a useful test for evaluating continuing response to levodopa therapy.

COMPONENTS OF MOTOR CONTROL ARE LINKED TO COGNITION IN PARKINSON'S DISEASE AND ARE RESISTANT TO TREATMENT

N Jordan, HJ Sagar, JA Cooper. Sheffield, UK

Components of motor control in Parkinson's disease (PD) may rest on different pathological mechanisms and respond differently to treatment. Cognitive and affective disturbances also occur in PD and clinical observations indicate an effect of mental state on motor control. Few studies, however, have formally examined these interactions. Accordingly, the authors analysed components of isotonic and isometric movement in $65 \mathrm{PD}$ patients. The experimental findings were related to the effects of specific treatment in a longitudinal study and to measures of cognition, depression and clinical motor disability. PD patients showed 1) parallel deficits in simple and choice motor reaction time. Motor reaction time correlated with frontal lobe function but not severity of depression; treatment did not improve performance; 2) impaired rates of force production and release. By contrast with motor reaction time, performance correlated with depression, but not cognition, and improved on treatment; 3) impaired ability to learn a motor skill, even when account was taken of weakness, incoordination and fatigue; skill learning showed no response to treatment. These results unequivocally demonstrate associations between cognition and components of motor control in PD which may be based on nondopaminergic pathology.

A STUDY OF 3,4-DIAMINOPYRIDINE (DAP) TREATMENT IN CONGENITAL (HEREDITARY) MYASTHENIA

JA Palace, $M$ Wiles, J Newsom-Davis, NMF Murray, KR Mills, A Vincent. University of Oxford and Institute of Neurology, UK

Congenital myasthenia (CM) describes a rare heterogeneous group of disorders affecting neuromuscular transmission. The defects can be pre-synaptic (deficiency of acetylcholine release) or post-synaptic (deficiency of acetylcholine receptors). There are no immunological abnormalities. Patients may respond to anticholinesterase medication. In an open prospective trial, the authors assessed the therapeutic effects of 3,4-DAP that increases transmitter release by blocking $\mathrm{K}^{+}$conductance at the nerve terminal, thereby prolonging the action potential.

Fourteen patients aged seven to 43 years, with clinical features of CM, were studied; seven had an affected sibling. All had responded to anticholinesterase medication and/or had electromyographic evidence of impaired neuromuscular transmission. The number of end-plate acetylcholine receptors $\left({ }^{125} \mathrm{I}\right.$-alphaBungarotoxin binding sites) in intercostal muscle at biopsy was reduced in six, normal in three, and not determined in the remainder. 3,4-DAP dosage ranged from $5-20 \mathrm{mg}$ orally, taken three to five times daily with or without pyridostigmine. Response was evaluated by performance scores, vital capacity and force measurements.

Based on the analysis of the initial worst three measurements, improvements were substantial in seven, and moderate in four. The principal side effects were perioral and distal paraesthesiae. It was concluded that 3,4-DAP alone or in combination with anticholinesterase medication may have a role in the treatment of congenital myasthenia.

SENSORY GATING BY MOVEMENT

W Schady, R Schmidt. Manchester, UK

There are complex interrelationships between motor commands and sensory feedback from the moved part. Movement alters cutaneous percepts and may attenuate somatosensory evoked potentials. The authors studied the effect of self-paced finger movements on sensations evoked by electrical stimulation of mechanoreceptive sensory units through microelectrodes placed in the median nerves of alert healthy adults. Stimulus intensity was set at threshold for conscious detection, resulting in a reproducible tactile sensation referred to the hand. Subjects were asked to rate the intensity of the intraneurally-evoked percept before, during and after movement of the finger to which the sensation was projected, or of a neighbouring finger.

The results of 39 experimental sessions in 10 subjects showed that finger movement inhibited sensation proportionately to finger speed. Suppression of cutaneous sensitivity was maximal in the moved finger but extended in a graded fashion to other fingers of the same hand. Local anaesthetic blocks of the median and other upper limb nerves counteracted most of the inhibitory effect of movement on cutaneous sensation. It is concluded that sensory gating is a focal and selective process which is largely mediated by cutaneous receptor input from the moving part. Its role is probably to prevent irrelevant information from reaching consciousness.

NEUROLOGICAL AND MRI ABNORMALITY IN PHENYLKETONURIA

AJ Thompson, I Smith, D Brenton, B Youl, G Rylance, DC Davidson, BE Kendall, AJ Lees. London, UK

Phenylketonuria (PKU) is a common autosomal recessive disorder resulting from a deficiency in the enzyme phenylalanine hydroxylase. Although the early development of severe neurological and intellectual impairment has been prevented by the introduction of a low phenylalanine diet (1962), the long term prognosis is still uncertain. Six patients were seen from three specialist centres in England with classical PKU who developed a neurological deficit in their late teens despite being on diet. There was a delay in diagnosis in three of the patients and four had intellectual impairment. Four patients developed a spastic quadraparesis and this was associated with ataxia in two, dystonia in one, sensory disturbance in one, and epilepsy in two. The neurological deficit developed gradually over six to 12 months and then remained stable. All six patients had abnormalities of the cerebral white matter on $T_{2}$ weighted MRI. These tended to be periventricular particularly involving the occipital lobe. Reinstitution of diet was beneficial in only two patients.

These findings have implications for the long term management of patients with PKU.

\section{HILLSBOROUGH-THE EPIDEMIOLOGY OF}

\section{ACUTE CEREBRAL ANOXIA}

The staff of the Sheffield Hospitals. Sheffield, UK

On 15 April 1989 over 90 people died in the Hillsborough football stadium. Twenty two patients were admitted to the Sheffield Hospitals with the consequences of acute cerebral anoxia.

Two patients did not recover and were declared brain dead within 48 hours. Five patients, having regained consciousness, became unresponsive, and developed seizures within two hours of the periud of anoxia.

Four patients were known to have had seizures on the football ground, a further 12 patients had seizures on arrival in the Accident and Emergency department, and five continued to have seizures over the next week.

Two patients remained in a vegetative state, four patients remained physically and cognitively handicapped, and two had persisting cortical blindness. The remainder made a rapid physical recovery, though half developed emotional problems. No cases of 
delayed post anoxic encephalopathy were seen.

Six patients had peripheral nerve injuries. Such an influx of acutely neurologically ill patients places a severe burden on local resources, both immediately and in the longer term.

ABNORMALITIES OF SACCADES IN PATIENTS WITH ESSENTIAL (IDIOPATHIC)

BLEPHAROSPASM

CJ Lueck, S Tanyeri, J Elston, C Kennard. London, UK

The aetiology of essential (idiopathic) blepharospasm is unclear. Whilst some clinicians consider it is purely psychogenic in origin, others believe it to be a form of adultonset torsion dystonia. The only pathophysiological evidence for the latter view comes from previously reported abnormalities in the blink reflex which suggest the pathology may lie in the basal ganglia. Blepharospasm also occurs as a feature of several other conditions involving the basal ganglia in which oculomotor abnormalities have also been observed. Analysis of saccadic eye movements was therefore performed in patients with the idiopathic form of the condition to search for further evidence of an underlying organic abnormality.

Seven patients (mean age 61 years) were compared with seven age-matched controls. Eye movements were recorded with the magnetic scleral search coil system, and saccades were analysed off-line using a digitising tablet. To generate saccades, subjects were instructed to follow a LED stepped randomly either horizontally or vertically.

Analyses of variance showed that patients saccadic latencies were statistically significantly longer than those of controls in both horizontal and downwards directions. Similarly, horizontal saccadic amplitude was reduced in the patient group, as were downward peak velocities. These results suggest an organic component to essential blepharospasm, the pathology of which also appears to involve the oculomotor system.

EXCITABILITY OF SINGLE MOTOR UNITS TO TRANSCRANIAL MAGNETIC STIMULATION OF THE BRAIN IN PARKINSON'S DISEASE JPR Dick, JR Baker, NR Davey, PH Ellaway, DW Maskill. London, UK

Primate experiments and clinical observation suggest that parkinsonian rigidity may be related to a small increase in the excitability of precentral and non-primary motor cortices. However, initial studies using high voltage electrical stimulation of the brain in the alert human subject failed to demonstrate such an increase of motor cortical excitability in Parkinson's disease.

Latency studies suggest that transcranial electrical stimulation excites corticomotoneurons directly and by-passes intracortical mechanisms. This limitation of electrical stimulation is not present with magnetic stimulation which is thought to excite corticomotoneurons transynaptically and hence may reveal the disturbance of motor cortical function thought to exist in Parkinson's disease.

The authors studied the probability of influencing the firing of a voluntarily recruited motor unit in the first dorsal interosseous muscle in response to a magnetic stimulus over the scalp. Using peri-stimulus time histograms the authors were able to compare the ease with which transynaptic activation of corticomotoneurons can be evoked in patients with Parkinson's disease $\mathrm{ON}$ and $\mathrm{OFF}$ therapy. Initial results support the notion that a modest motor cortical hyperexcitability exists in patients with Parkinson's disease when OFF therapy. 